\title{
Assortative mixing in networks
}

\author{
M. E. J. Newman \\ Department of Physics, University of Michigan, Ann Arbor, MI 48109-1120 and \\ Santa Fe Institute, 1399 Hyde Park Road, Santa Fe, NM 87501
}

\begin{abstract}
A network is said to show assortative mixing if the nodes in the network that have many connections tend to be connected to other nodes with many connections. We define a measure of assortative mixing for networks and use it to show that social networks are often assortatively mixed, but that technological and biological networks tend to be disassortative. We propose a model of an assortative network, which we study both analytically and numerically. Within the framework of this model we find that assortative networks tend to percolate more easily than their disassortative counterparts and that they are also more robust to vertex removal.
\end{abstract}

Many systems take the form of networks - sets of vertices joined together by edges - including social networks, computer networks, and biological networks [1, 2, 3]. A variety of models of networks have been proposed and studied in the physics literature, many of which have been successful at reproducing features of networks in the real world [4, 5, 6. One particularly well-studied model is the cumulative advantage or preferential attachment model [6, 6, 8, 9, 10] in which the probability of a given source vertex forming a connection to a target vertex is some (usually increasing) function of the degree of the target vertex. (The degree of a vertex is the number of other vertices to which it is attached.) Preferential attachment processes are widely accepted as the probable explanation for the power-law and other skewed degree distributions seen in many networks 11, 12, 13, 14.

However, there is an important element missing from these as well as other network models: in none of these models does the probability of attachment to the target vertex depend also on the degree of the source vertex. In the real world on the other hand such dependencies are common. Many networks show "assortative mixing" on their degrees, i.e., a preference for high-degree vertices to attach to other high-degree vertices. Others show disassortative mixing - high-degree vertices attach to lowdegree ones. In this paper we first demonstrate the presence of assortative mixing in a variety of networks by direct measurement, and then argue, using exactly solvable models and numerical simulations, that assortative mixing can have a substantial effect on the behavior of networked systems. Models that do not take it into account will necessarily fail to reproduce correctly many of the behaviors of real-world networked systems.

Consider then a network, represented in the simplest case by an undirected graph of $N$ vertices and $M$ edges, with degree distribution $p_{k}$. That is, $p_{k}$ is the probability that a randomly chosen vertex on the graph will have degree $k$. Now consider a vertex reached by following a randomly chosen edge on the graph. The degree of this vertex is not distributed according to $p_{k}$. Instead it is biased in favor of vertices of high degree, since more edges end at a high-degree vertex than at a low-degree one. This means that the degree distribution for the vertex at the end of a randomly chosen edge is proportional $k p_{k}$, rather than just $p_{k}$. In this paper, we will usually be interested not in the total degree of such a vertex, but in the remaining degree - the number of edges leaving the vertex other than the one we arrived along. This number is one less than the total degree and hence is distributed in proportion to $(k+1) p_{k+1}$. The correctly normalized distribution $q_{k}$ of the remaining degree is then

$$
q_{k}=\frac{(k+1) p_{k+1}}{\sum_{j} j p_{j}}
$$

Following Callaway et al. [15], we now define the quantity $e_{j k}$ to be the joint probability distribution of the remaining degrees of the two vertices at either end of a randomly chosen edge [34]. This quantity is symmetric in its indices on an undirected graph $e_{j k}=e_{k j}$, and obeys the sum rules

$$
\sum_{j k} e_{j k}=1, \quad \sum_{j} e_{j k}=q_{k}
$$

In a network with no assortative (or disassortative) mixing $e_{j k}$ takes the value $q_{j} q_{k}$. If there is assortative mixing, $e_{j k}$ will differ from this value and the amount of assortative mixing can be quantified by the connected degree-degree correlation function $\langle j k\rangle-\langle j\rangle\langle k\rangle=$ $\sum_{j k} j k\left(e_{j k}-q_{j} q_{k}\right)$, where $\langle\ldots\rangle$ indicates an average over edges [15]. This correlation function is zero for no assortative mixing and positive or negative for assortative or disassortative mixing respectively. For the purposes of comparing different networks, it is convenient to normalize it by dividing by its maximal value, which it achieves on a perfectly assortative network, i.e., one with $e_{j k}=q_{k} \delta_{j k}$. This value is equal to the variance $\sigma_{q}^{2}=\sum_{k} k^{2} q_{k}-\left[\sum_{k} k q_{k}\right]^{2}$ of the distribution $q_{k}$, and hence the normalized correlation function is

$$
r=\frac{1}{\sigma_{q}^{2}} \sum_{j k} j k\left(e_{j k}-q_{j} q_{k}\right),
$$

which is simply the Pearson correlation coefficient of the degrees at either ends of an edge and lies in the range $-1 \leq r \leq 1$ 35. For the practical purpose of evaluating $r$ on an observed network, we can rewrite (3) as

$$
r=\frac{M^{-1} \sum_{i} j_{i} k_{i}-\left[M^{-1} \sum_{i} \frac{1}{2}\left(j_{i}+k_{i}\right)\right]^{2}}{M^{-1} \sum_{i} \frac{1}{2}\left(j_{i}^{2}+k_{i}^{2}\right)-\left[M^{-1} \sum_{i} \frac{1}{2}\left(j_{i}+k_{i}\right)\right]^{2}},
$$




\begin{tabular}{|c|c|c|c|}
\hline & network & $n$ & $r$ \\
\hline \multirow{9}{*}{ 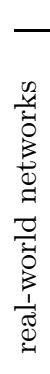 } & physics coauthorship $^{a}$ & 52909 & 0.363 \\
\hline & biology coauthorship $^{\mathrm{a}}$ & 1520251 & 0.127 \\
\hline & mathematics coauthorship ${ }^{\mathrm{b}}$ & 253339 & 0.120 \\
\hline & film actor collaborations ${ }^{\mathrm{c}}$ & 449913 & 0.208 \\
\hline & company directors $^{\mathrm{d}}$ & 7673 & 0.276 \\
\hline & Internet $^{\mathrm{e}}$ & 10697 & -0.189 \\
\hline & World-Wide Web ${ }^{\mathrm{f}}$ & 269504 & -0.065 \\
\hline & protein interactions ${ }^{\mathrm{g}}$ & 2115 & -0.156 \\
\hline & neural network $^{\mathrm{h}}$ & 307 & -0.163 \\
\hline & food web ${ }^{i}$ & 92 & -0.276 \\
\hline & random graph ${ }^{\mathrm{u}}$ & & \\
\hline סू & Callaway et al. ${ }^{\mathrm{v}}$ & & $\delta /(1+2 \delta)$ \\
\hline & Barabási and Albert ${ }^{w}$ & & \\
\hline
\end{tabular}

TABLE I: Size $n$ and assortativity coefficient $r$ for a number of different networks: collaboration networks of (a) scientists in physics and biology [16], (b) mathematicians [17], (c) film actors 《4, and (d) businesspeople [18]; (e) connections between autonomous systems on the Internet [19]; (f) undirected hyperlinks between Web pages in a single domain 6 ; (g) protein-protein interaction network in yeast 20; (h) undirected (and unweighted) synaptic connections in the neural network of the nematode $C$. Elegans [4]; (i) undirected trophic relations in the food web of Little Rock Lake, Wisconsin [21]. The last three lines give analytic results for model networks in the limit of large network size: (u) the random graph of Erdös and Rényi [22]; (v) the grown graph model of Callaway et al. [15]; (w) the preferential attachment model of Barabási and Albert [6].

where $j_{i}, k_{i}$ are the degrees of the vertices at the ends of the $i$ th edge, with $i=1 \ldots M$ [36].

In Table we show values of $r$ for a variety of real-world networks. As the table shows, of the social networks studied (the top five entries in the table) all have significant assortative mixing, which accords with accepted wisdom within the sociological community. By contrast, the technological and biological networks studied (the middle five entries) all have disassortative mixing - high degree vertices preferentially connect with low degree ones and vice versa. Various explanations for this observation suggest themselves. In the case of the Internet, for example, it appears that the high degree vertices mostly represent connectivity providers - telephone companies and other communications carriers-who typically have a large number of connections to clients who themselves have only a single connection [19]. Thus the high-degree vertices do indeed tend to be connected to the low-degree ones.

We have also calculated $r$ analytically for three models of networks: (1) the random graph of Erdős and Rényi [22], in which edges are placed at random between a fixed set of vertices; (2) the grown graph model of Callaway et al. [15], in which both edges and vertices are added at random at constant but possibly different rates, the ratio of the rates being denoted $\delta ;(3)$ the grown graph model of Barabási and Albert [6], in which both edges and vertices are added, and one end of each edge is added with linear preferential attachment.
For the random graph, since edges are placed at random without regard to vertex degree it follows trivially that $r=0$ in the limit of large graph size. The model of Callaway et al. however, although apparently similar in construction, gives a markedly different result. From Eq. (21) of Ref. 15, $e_{j k}$ for this model satisfies the recurrence relation

$$
(1+4 \delta) e_{j k}=2 \delta\left(e_{j-1, k}+e_{j, k-1}\right)+p_{j} p_{k}
$$

and the degree distribution is $p_{k}=(2 \delta)^{k} /(1+2 \delta)^{k+1}$. Substituting into Eq. (3) and making use of Eq. (2), we then find that $r=\delta /(1+2 \delta)$. Thus the model shows significant assortative mixing, with a maximum value of $r=\frac{1}{2}$ in the limit of large $\delta$. This agrees with intuition [15]: in the grown graph the older vertices have higher degree and also tend to have higher probability of being connected to one another, simply by virtue of being around for longer. Thus one would expect positive assortative mixing.

The model of Barabási and Albert [6] provides an interesting counter-example to this intuition. Although this is a grown graph model, in which again older vertices have higher degree 23, it shows no assortative mixing at all. Making use of Eq. (42) of Ref. 24 we can show that $e_{j k}$ for the model of Barabási and Albert goes asymptotically as $1 /\left(j^{2} k^{2}\right)-6 /(j+k)^{4}$ in the limit of large $j$ and $k$, which implies that $r \rightarrow 0$ as $\left(\log ^{2} N\right) / N$ as $N$ becomes large. The model of Barabási and Albert has been used as a model of the structure of the Internet and the World-Wide Web. Since these networks show significant disassortative mixing however (Table $\mathbb{1}$ ), it is clear that the model is incomplete. It is an interesting open question what type of network evolution processes could explain the values of $r$ observed in real-world networks.

Turning now to theoretical developments, we propose a simple model of an assortatively mixed network, which is exactly solvable for many of its properties in the limit of large graph size. Consider the ensemble of graphs in which the distribution $e_{j k}$ takes a specified value. This defines a random graph model similar in concept to the random graphs with specified degree sequence [5, 25, 26], except for the added element of assortative mixing.

Consider a typical member of this ensemble in the limit of large graph size, and consider a randomly chosen edge in that graph, one end of which is attached to a vertex of degree $j$. We ask what the probability distribution is of the number of other vertices reachable by following that edge. Let this probability distribution be generated by a generating function $G_{j}(x)$, which depends in general on the degree $j$ of the starting vertex. By arguments similar to those of Ref. 5 , we can show that $G_{j}(x)$ must satisfy a self-consistency condition of the form

$$
G_{j}(x)=x \frac{\sum_{k} e_{j k}\left[G_{k}(x)\right]^{k}}{\sum_{k} e_{j k}},
$$

while the number of vertices reachable from a randomly 
chosen vertex is generated by

$$
H(x)=x p_{0}+x \sum_{k=1}^{\infty} p_{k}\left[G_{k-1}(x)\right]^{k} .
$$

The average size of the component to which such a vertex belongs is given by the derivative of $H:\langle s\rangle=H^{\prime}(1)=$ $1+\sum_{k} k p_{k} G_{k-1}^{\prime}(1)$. Differentiating Eq. (6) we then get

$$
\langle s\rangle=1-z \mathbf{q} \cdot \mathbf{A}^{-1} \cdot \mathbf{q},
$$

where $z$ is the mean degree, $\mathbf{q}$ is the vector whose elements are the $q_{k}$, and $\mathbf{A}$ is the asymmetric matrix with elements $A_{j k}=k e_{j k}-q_{k} \delta_{j k}$.

Equation (8) diverges at the point at which the determinant of $\mathbf{A}$ is zero. This point marks the phase transition at which a giant component forms in our graph. By considering the behavior of Eq. (8) close to the transition, where $\langle s\rangle$ must be large and positive in the absence of a giant component, we deduce that a giant component exists in the network when $\operatorname{det} \mathbf{A}>0$. This is the appropriate generalization for a network with assortative mixing of the criterion of Molloy and Reed 26] for the existence of a giant component.

To calculate the size $S$ of the giant component, we define $u_{k}$ to be the probability that an edge connected to a vertex of remaining degree $k$ leads to another vertex that does not belong to the giant component. Then

$$
S=1-p_{0}-\sum_{k=1}^{\infty} p_{k} u_{k-1}^{k}, \quad u_{j}=\frac{\sum_{k} e_{j k} u_{k}^{k}}{\sum_{k} e_{j k}} .
$$

As with most other random graph models, including the original model of Erdős and Rényi, it is usually not possible to solve for $S$ in closed form, but we can determine it by numerical iteration from a suitable set of starting values for $u_{k}$.

To test these results and to help form a more complete picture of the properties of assortatively mixed networks, we have also performed computer simulations, generating networks with given values of $e_{j k}$ and measuring their properties directly. Generating such networks is not entirely trivial. One cannot simply draw a set of degree pairs $\left(j_{i}, k_{i}\right)$ for edges $i$ from the distribution $e_{j k}$, since such a set would almost certainly fail to satisfy the basic topological requirement that the number of edges ending at vertices of degree $k$ must be a multiple of $k$. Instead therefore we propose the following Monte Carlo algorithm for generating graphs.

First, we generate a random graph with the desired degree distribution according to the prescription given in Ref. 26. Then we apply a Metropolis dynamics to the graph in which on each step we choose at random two edges, denoted by the vertex pairs, $\left(v_{1}, w_{1}\right)$ and $\left(v_{2}, w_{2}\right)$, that they connect. We measure the remaining degrees $\left(j_{1}, k_{1}\right)$ and $\left(j_{2}, k_{2}\right)$ for these vertex pairs, and then replace the edges with two new ones $\left(v_{1}, v_{2}\right)$ and $\left(w_{1}, w_{2}\right)$ with probability $\min \left(1,\left(e_{j_{1} j_{2}} e_{k_{1} k_{2}}\right) /\left(e_{j_{1} k_{1}} e_{j_{2} k_{2}}\right)\right)$. This dynamics conserves the degree sequence, is ergodic on the set of graphs having that degree sequence, and, with the choice of acceptance probability above, satisfies detailed balance for state probabilities $\prod_{i} e_{j_{i} k_{i}}$, and hence has the required edge distribution $e_{j k}$ as its fixed point.

As an example, consider the symmetric binomial form

$$
e_{j k}=\mathcal{N} \mathrm{e}^{-(j+k) / \kappa}\left[\left(\begin{array}{c}
j+k \\
j
\end{array}\right) p^{j} q^{k}+\left(\begin{array}{c}
j+k \\
k
\end{array}\right) p^{k} q^{j}\right],
$$

where $p+q=1, \kappa>0$, and $\mathcal{N}=\frac{1}{2}\left(1-\mathrm{e}^{-1 / \kappa}\right)$ is a normalizing constant. (The binomial probabilities $p$ and $q$ should not be confused with the quantities $p_{k}$ and $q_{k}$ introduced earlier.) This distribution is chosen for analytic tractability, although its behavior is also quite natural: the distribution of the sum $j+k$ of the degrees at the ends of an edge falls off as a simple exponential, while that sum is distributed between the two ends binomially, the parameter $p$ controlling the assortative mixing. From Eq. (3), the value of $r$ is

$$
r=\frac{8 p q-1}{2 \mathrm{e}^{1 / \kappa}-1+2(p-q)^{2}},
$$

which can take both positive and negative values, passing through zero when $p=p_{0}=\frac{1}{2}-\frac{1}{4} \sqrt{2}=0.1464 \ldots$

In Fig. 1 we show the size of the giant component for graphs of this type as a function of the degree scale parameter $\kappa$, from both our numerical simulations and the exact solution above. As the figure shows, the two are in good agreement. The three curves in the figure are for $p=0.05$, where the graph is disassortative, $p=p_{0}$, where it is neutral (neither assortative nor disassortative), and $p=0.5$, where it is assortative.

As $\kappa$ becomes large we see the expected phase transition at which a giant component forms. There are two important points to notice about the figure. First, the position of the phase transition moves lower as the graph becomes more assortative. That is, the graph percolates more easily, creating a giant component, if the highdegree vertices preferentially associate with other highdegree ones. Second, notice that, by contrast, the size of the giant component for large $\kappa$ is smaller in the assortatively mixed network.

These findings are intuitively reasonable. If the network mixes assortatively, then the high-degree vertices will tend to stick together in a subnetwork or core group of higher mean degree than the network as a whole. It is reasonable to suppose that percolation would occur earlier within such a subnetwork. Conversely, since percolation will be restricted to this subnetwork, it is not surprising that the giant component has a smaller size in this case than when the network is disassortative. These results could have implications, for example, for the spread of disease on social networks [27] - social networks being assortatively mixed in many cases, as Table 1 shows. The core group of an assortatively mixed network could form a "reservoir" for disease, sustaining an epidemic even in cases in which the network is not sufficiently dense on average for the disease to persist. On the other hand, one 


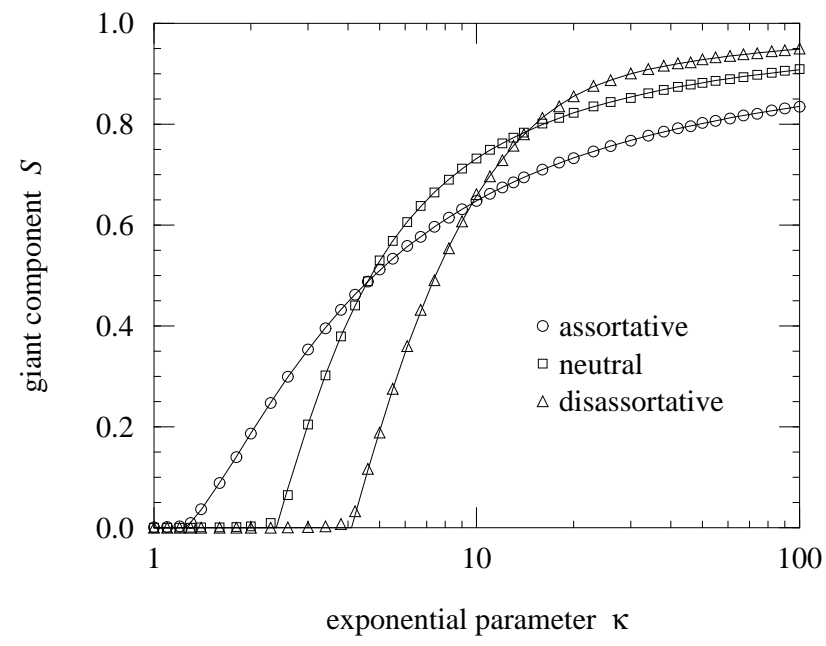

FIG. 1: Size of the giant component as a fraction of graph size for graphs with the edge distribution given in Eq. (10). The points are simulation results for graphs of $N=100000$ vertices while the solid lines are the numerical solution of Eq. (9). Each point is an average over ten graphs; the resulting statistical errors are smaller than the symbols. The values of $p$ are 0.5 (circles), $p_{0}=0.146 \ldots$ (squares), and 0.05 (triangles).

would expect the disease to be restricted to a smaller segment of the population in such cases than for diseases spreading on neutral or disassortative networks.

Assortative mixing also has implications for questions of network resilience, the subject of much discussion in the recent literature 28, 29, 30, 31, 32. It has been found that the connectivity of many networks (i.e., the existence of paths between pairs of vertices) can be destroyed by the removal of just a few of the highest degree vertices, a result that may have applications in, for example, vaccination strategies [33]. In assortatively mixed networks, however, we find numerically that removing high-degree vertices is a relatively inefficient strategy for destroying network connectivity, presumably because these vertices tend to be clustered together in the core group, so that removing them is somewhat redundant. In a disassortative network with a similarly sized giant component attacks on the highest degree vertices are much more effective, these vertices being broadly distributed over the network and presumably therefore forming links on many paths between other vertices. For networks of the type described by Eq. (10) we find that the number of high-degree vertices that need to be removed to destroy similarly sized giant components is greater by a factor of about five to ten in an assortative network $(p=0.5)$ than in a disassortative one $(p=0.05)$ for the typical parameter values studied here.

These considerations paint rather a grim picture: the networks that we might want to break up, such as the social networks that spread disease, appear to be assortative, and therefore are resilient, at least against simple targeted attacks such as attacks on the highest degree vertices. And yet at the same time the networks that we would wish to protect, including technological networks such as the Internet, appear to be disassortative, and are hence particularly vulnerable.

To conclude, in this paper we have studied assortative mixing by degree in networks - the tendency for highdegree vertices to associate preferentially with other highdegree vertices. We have defined a scalar measure of assortative mixing and used it to show that many social networks have significant assortative mixing, while technological and biological networks seem to be disassortative. We have also proposed a model of an assortatively mixed network, which we have solved exactly using generating function techniques, and also simulated using a Monte Carlo graph sampling method. Within this model we find that assortative networks percolate more easily and that they are also more robust to removal of their highest degree vertices, while disassortative networks percolate less easily and are more vulnerable. This suggests that social networks may be robust to intervention and attack while technological networks are not.

The author thanks Duncan Callaway, Michelle Girvan, Cris Moore, and Martina Morris for helpful comments, and László Barabási, Jerry Davis, Jerry Grossman, Hawoong Jeong, Neo Martinez, and Duncan Watts for providing network data used in the calculations for Table 1 . This work was funded in part by the National Science Foundation under grant DMS-0109086.
[1] S. H. Strogatz, Exploring complex networks. Nature 410, 268-276 (2001).

[2] R. Albert and A.-L. Barabási, Statistical mechanics of complex networks. Rev. Mod. Phys. 74, 47-97 (2002).

[3] S. N. Dorogovtsev and J. F. F. Mendes, Evolution of networks. Advances in Physics 51, 1079 (2002).

[4] D. J. Watts and S. H. Strogatz, Collective dynamics of 'small-world' networks. Nature 393, 440-442 (1998).

[5] M. E. J. Newman, S. H. Strogatz, and D. J. Watts, Random graphs with arbitrary degree distributions and their applications. Phys. Rev. E 64, 026118 (2001).

[6] A.-L. Barabási and R. Albert, Emergence of scaling in random networks. Science 286, 509-512 (1999).

[7] H. A. Simon, On a class of skew distribution functions. Biometrika 42, 425-440 (1955).

[8] D. J. de S. Price, A general theory of bibliometric and other cumulative advantage processes. J. Amer. Soc. Inform. Sci. 27, 292-306 (1976).

[9] P. L. Krapivsky, S. Redner, and F. Leyvraz, Connectivity of growing random networks. Phys. Rev. Lett. 85, 46294632 (2000).

[10] S. N. Dorogovtsev, J. F. F. Mendes, and A. N. Samukhin, Structure of growing networks with preferential linking. Phys. Rev. Lett. 85, 4633-4636 (2000). 
[11] D. J. de S. Price, Networks of scientific papers. Science 149, 510-515 (1965).

[12] R. Albert, H. Jeong, and A.-L. Barabási, Diameter of the world-wide web. Nature 401, 130-131 (1999).

[13] M. Faloutsos, P. Faloutsos, and C. Faloutsos, On powerlaw relationships of the internet topology. Computer Communications Review 29, 251-262 (1999).

[14] L. A. N. Amaral, A. Scala, M. Barthélémy, and H. E. Stanley, Classes of small-world networks. Proc. Natl. Acad. Sci. USA 97, 11149-11152 (2000).

[15] D. S. Callaway, J. E. Hopcroft, J. M. Kleinberg, M. E. J. Newman, and S. H. Strogatz, Are randomly grown graphs really random? Phys. Rev. E 64, 041902 (2001).

[16] M. E. J. Newman, The structure of scientific collaboration networks. Proc. Natl. Acad. Sci. USA 98, 404-409 (2001).

[17] J. W. Grossman and P. D. F. Ion, On a portion of the well-known collaboration graph. Congressus Numerantium 108, 129-131 (1995).

[18] G. F. Davis, M. Yoo, and W. E. Baker, The small world of the corporate elite. Preprint, University of Michigan Business School (2001).

[19] Q. Chen, H. Chang, R. Govindan, S. Jamin, S. J. Shenker, and W. Willinger, The origin of power laws in Internet topologies revisited. In Proceedings of the 21st Annual Joint Conference of the IEEE Computer and Communications Societies, IEEE Computer Society (2002).

[20] H. Jeong, S. Mason, A.-L. Barabási, and Z. N. Oltvai, Lethality and centrality in protein networks. Nature 411, 41-42 (2001).

[21] N. D. Martinez, Artifacts or atributes? Effects of resolution on the Little Rock Lake food web. Ecological Monographs 61, 367-392 (1991).

[22] B. Bollobás, Random Graphs. Academic Press, New York, 2nd edition (2001).

[23] L. A. Adamic and B. A. Huberman, Power-law distribution of the world wide web. Science 287, 2115a (2000).

[24] P. L. Krapivsky and S. Redner, Organization of growing random networks. Phys. Rev. E 63, 066123 (2001).

[25] E. A. Bender and E. R. Canfield, The asymptotic number of labeled graphs with given degree sequences. Journal of
Combinatorial Theory A 24, 296-307 (1978).

[26] M. Molloy and B. Reed, A critical point for random graphs with a given degree sequence. Random Structures and Algorithms 6, 161-179 (1995).

[27] M. Morris, Telling tails explain the discrepancy in sexual partner reports. Nature 365, 437-440 (1993).

[28] R. Albert, H. Jeong, and A.-L. Barabási, Attack and error tolerance of complex networks. Nature 406, 378382 (2000).

[29] R. Cohen, K. Erez, D. ben-Avraham, and S. Havlin, Resilience of the Internet to random breakdowns. Phys. Rev. Lett. 85, 4626-4628 (2000).

[30] R. Cohen, K. Erez, D. ben-Avraham, and S. Havlin, Breakdown of the Internet under intentional attack. Phys. Rev. Lett. 86, 3682-3685 (2001).

[31] D. S. Callaway, M. E. J. Newman, S. H. Strogatz, and D. J. Watts, Network robustness and fragility: Percolation on random graphs. Phys. Rev. Lett. 85, 5468-5471 (2000).

[32] P. Holme, B. J. Kim, C. N. Yoon, and S. K. Han, Attack vulnerability of complex networks. Phys. Rev. E 65, 056109 (2002).

[33] R. Pastor-Satorras and A. Vespignani, Immunization of complex networks. Phys. Rev. E 65, 036104 (2002).

[34] A related quantity has been studied by Krapivsky and Redner 24] in the context of the model of Barabási and Albert [6]. That quantity however, which is denoted $n_{k l}$, is more complex than the one used here, being asymmetric in its indices, because one index is designated as being the "ancestral" index with respect to the order in which the graph was grown.

[35] The quantity $r$ can easily be generalized to the case of a directed network, where $e_{j k}$ is asymmetric and $r=$ $\sum_{j k} j k\left(e_{j k}-q_{j} q_{k}\right) /\left(\sigma_{\text {in }} \sigma_{\text {out }}\right)$, with $\sigma_{\text {in }}$ and $\sigma_{\text {out }}$ being the standard deviations of the remaining degrees at the in-going and out-going ends of the edge respectively.

[36] One can use either the total degrees or the remaining degrees to evaluate Eq. (四) - the answer is the same either way. Note also that we have written Eq. (4) in a form manifestly symmetric in $j_{i}$ and $k_{i}$, so that it doesn't matter which end of an edge is which. 